\title{
Synchronously Multiple Gastric Adenocarcinomas with Intestinal Mucin Phenotype in a Patient not Infected with Helicobacter pylori, Showing a Gastritis-like Appearance
}

\author{
Kenichi Kishimoto ${ }^{1,2}$, Kotaro Shibagaki ${ }^{1}$, Ayako Itawaki ${ }^{1}$, Shinsaku Tanaka ${ }^{2}$, \\ Yusuke Takahashi ${ }^{2}$, Yumi $\mathrm{Cho}^{2}$, Yukihiro Ikuta ${ }^{2}$, Naoki Oshima ${ }^{2}$, \\ Makoto Nagasaki ${ }^{3}$ and Shunji Ishihara ${ }^{1}$
}

\begin{abstract}
:
Helicobacter pylori (H. pylori)-negative gastric cancer (HPNGC) usually shows a gastric mucin phenotype, but there are a few case reports of HPNGC with an intestinal mucin phenotype. We herein report a case of multiple HPNGC with an intestinal mucin phenotype showing a gastritis-like appearance. A 68-year-old $H$. pylori-uninfected man was suspected of having antral gastritis on endoscopy, but a histologic examination revealed multiple well-differentiated adenocarcinomas with positive-CDX2/MUC2/CD10 and negative-MUC5 AC/MUC6. P53 was overexpressed, and intestinal metaplasia was sporadically detected in the non-atrophic mucosal background, thus indicating $H$. pylori-unrelated multistage carcinogenesis. The neoplastic surfaces were covered by a non-neoplastic epithelium, which caused a gastritis-like appearance. This report suggested the possibility of overlooking this neoplasm.
\end{abstract}

Key words: Helicobacter pylori, uninfected, gastric cancer, intestinal mucin, intestinal metaplasia

(Intern Med 59: 3155-3159, 2020)

(DOI: 10.2169/internalmedicine.5393-20)

\section{Introduction}

Gastric cancer is caused primarily by a chronic Helicobacter pylori ( $H$. pylori) infection that leads to atrophic gastritis with intestinal metaplasia. H. pylori-negative gastric cancer (HPNGC) is reported to represent $0.42-0.66 \%$ of all gastric cancer cases $(1,2)$. The majority of HPNGCs are reportedly signet-ring cell carcinomas; however, case series of differentiated adenocarcinomas, including fundic gland-type and foveolar-type, have recently been published $(3,4)$. These HPNGCs reportedly express a gastric mucin phenotype, as they occur in the normal gastric mucosa in the absence of intestinal metaplasia. However, we recently encountered some case reports of a solitary HPNGC with an intestinal mucin phenotype (5-7), although little information regarding their clinicopathological characteristics was pro- vided. They were all reported to be solitary lesions in nonatrophic pyloric gland mucosa; multiple simultaneous lesions have not been reported thus far. We herein report the first known case of multiple HPNGCs with an intestinal mucin phenotype displaying a gastritis-like appearance.

\section{Case Report}

A 68-year-old man underwent screening for esophagogastroduodenoscopy (EGD), and three reddish depressed lesions were observed in a non-atrophic pyloric gland mucosa, closely resembling superficial gastritis on white-light endoscopy (WLE). Narrow-band imaging with magnification endoscopy (NBIME) revealed slightly irregularly shaped papillary or tubular microstructures with irregularly dilated microvessels that were either unclearly demarcated from the surrounding mucosa or partially covered by non-neoplastic

${ }^{1}$ Department of Gastroenterology, Faculty of Medicine, Shimane University, Japan, ${ }^{2}$ Department of Gastroenterology, National Hospital Organization Hamada Medical Center, Japan and ${ }^{3}$ Department of Pathology, National Hospital Organization Hamada Medical Center, Japan Received: May 20, 2020; Accepted: June 7, 2020; Advance Publication by J-STAGE: July 21, 2020

Correspondence to Dr. Kotaro Shibagaki, kotaro@med.shimane-u.ac.jp 

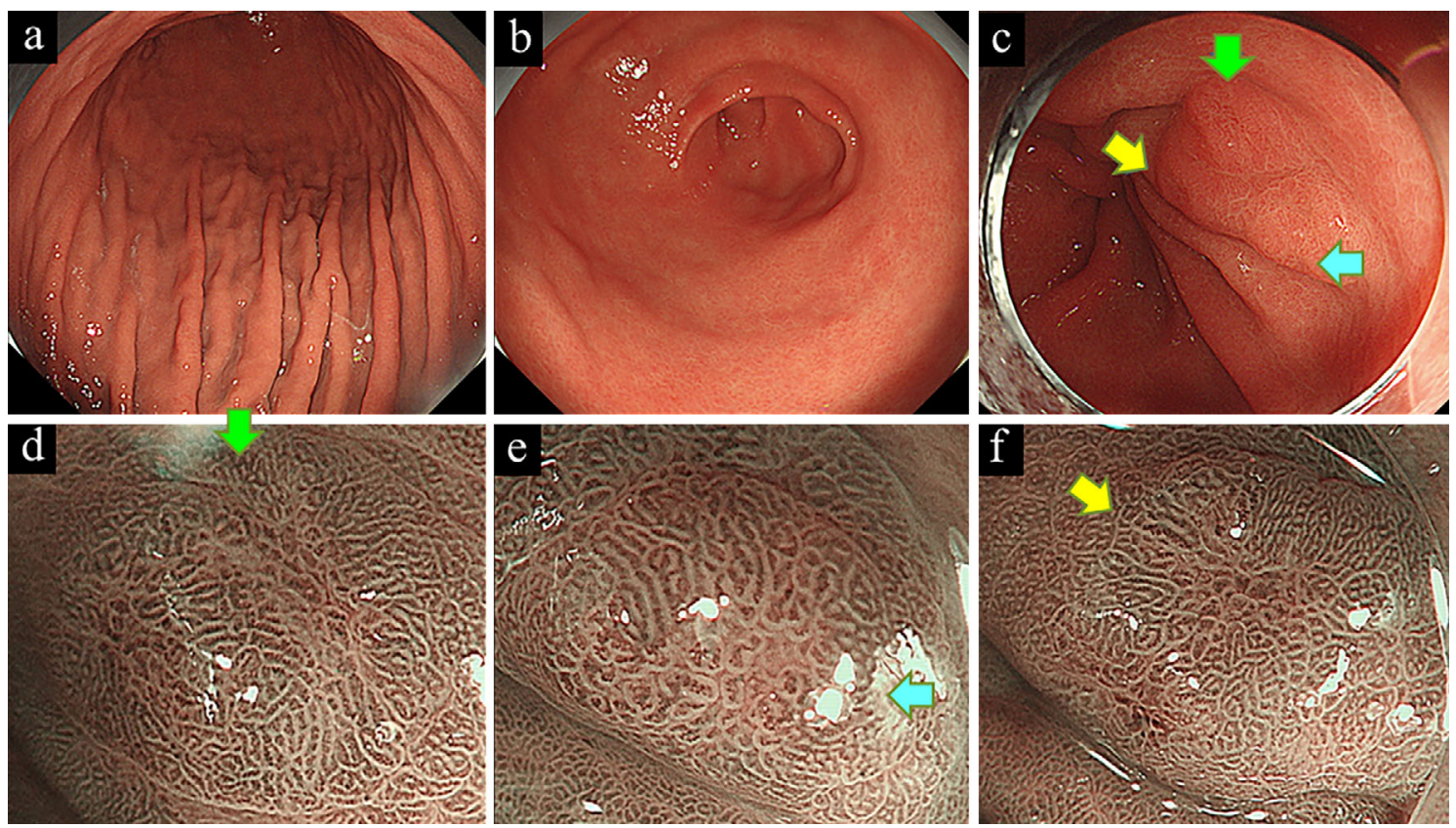

Figure 1. White-light endoscopy showed a non-atrophic gastric mucosa of the gastric body (a) and antrum (b), suggesting an Helicobacter pylori-uninfected stomach. Three depressed lesions (arrows) were observed in the prepyloric area, showing a gastritis-like appearance (c). Narrow-band imaging with magnification endoscopy showed slightly irregularly shaped papillary or tubular microstructures that were either unclearly demarcated from the surrounding mucosa or partially covered by a non-neoplastic epithelium (d-f).

epithelium, giving the impression of inflammation rather than neoplastic lesions (Fig. 1). However, a histologic examination of the biopsy specimen revealed welldifferentiated tubular adenocarcinoma. The patient did not have family history of gastric cancer or any other associated malignant tumors associated with other hereditary tumor syndromes, such as familial adenomatous polyposis or Lynch syndrome, et cetera. He currently was a smoker and also regularly drank alcohol.

The patient had never received $H$. pylori eradication therapy. His serum anti- $H$. pylori $\mathrm{IgG}$ antibody titer was $<3 \mathrm{U} /$ $\mathrm{mL}$ and ${ }^{13} \mathrm{C}$-labeled urea breath test value was $<2.5 \%$. The background mucosa was diagnosed as a non-atrophic pyloric gland mucosa based on the Kimura Takemoto classification (8) for endoscopic findings. The antral and body mucosa revealed slight redness and mild edematous change, respectively, but they were diagnosed to be within the normal range. Consequently, the neoplasms were considered to have arisen synchronously from an $H$. pylori-uninfected gastric mucosa, and they were simultaneously resected by endoscopic submucosal dissection.

The resected specimens showed three slightly depressed lesions. A histologic examination revealed irregularly shaped neoplastic glands with roundish or polygonal nuclei lacking polarity that were clearly demarcated from the adjoining foveolar epithelium. The neoplastic surface was partially covered by a non-neoplastic foveolar epithelium in two of the three lesions (Fig. 2). None of the neoplasms showed either stromal or lymphovascular invasion. However, an immuno- histochemical analysis revealed a Ki-67 overexpression (labeling index, 65.2\%) and p53 overstaining for the neoplastic cells. The neoplastic cells were positive for CDX2/MUC2/ CD10 and negative for MUC5AC/MUC6, thus suggesting a pure intestinal mucin phenotype (Fig. 3). All neoplasms were diagnosed to be noninvasive low/high-grade neoplasia according to the Padova/Vienna classification, as low/highgrade intraepithelial dysplasia by the World Health Organization (WHO) classification, and as differentiated adenocarcinoma in the Japanese Classification of Gastric Carcinoma. The background mucosa histologically showed little inflammatory reaction (neutrophils; normal and mononuclear cell; mild), no mucosal atrophy, and no spirillum including $H$. pylori or non-Helicobacter pylori Helicobacter based on the updated Sydney system (9), but intestinal metaplasia was sporadically detected in the prepyloric area, which was later proven by a histopathologic examination of the biopsy specimen (Fig. 4).

\section{Discussion}

HPNGC has been previously reported to represent $<1 \%$ of all gastric cancers $(1,2)$, but the incidence is strongly expected to increase in Japan in the future based on a sharp decrease in the $H$. pylori-infected population (10). The majority of HPNGC cases show a gastric mucin phenotype, as they occur in $H$. pylori-uninfected gastric mucosa without intestinal metaplasia. Consequently, to understand the occurrence of HPNGC with an intestinal mucin phenotype, proof 


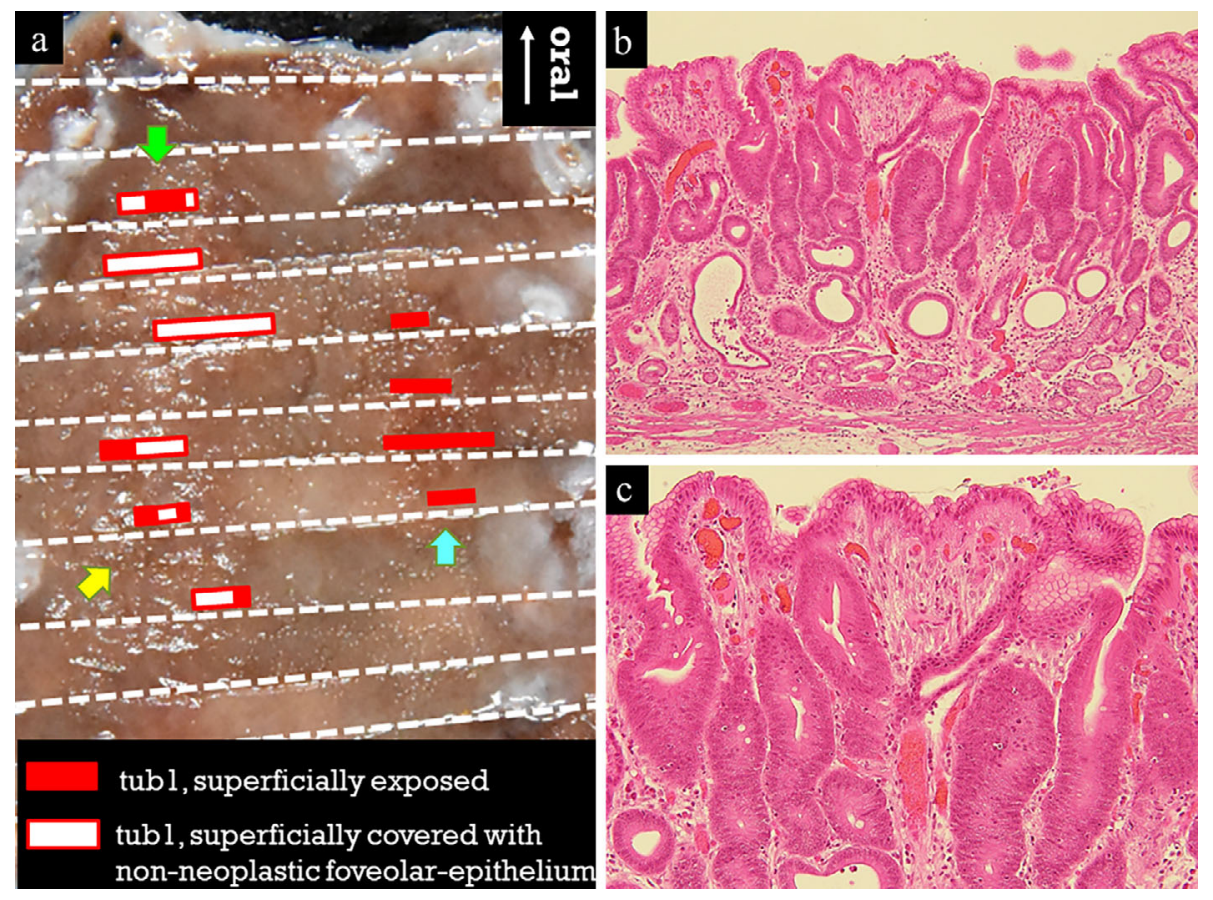

Figure 2. Histologic mapping of the resected specimen showed three mucosal cancers. The red square indicates superficially exposed tubular adenocarcinoma, and the white square with red border indicates tubular adenocarcinoma superficially covered with a non-neoplastic foveolar-epithelium (a). In the lesion indicated by the green arrow, an irregularly shaped tubular neoplastic duct was confirmed in the middle layer of the lamina propria (b). The neoplastic cells had either roundish or polygonal nuclei lacking polarity, and the lesion surface was partially covered by a non-neoplastic foveolar epithelium (c). Only slight stromal inflammation was observed, and no spirillum was detected, thus suggesting the presence of a Helicobacter pylori-uninfected pyloric gland mucosa.
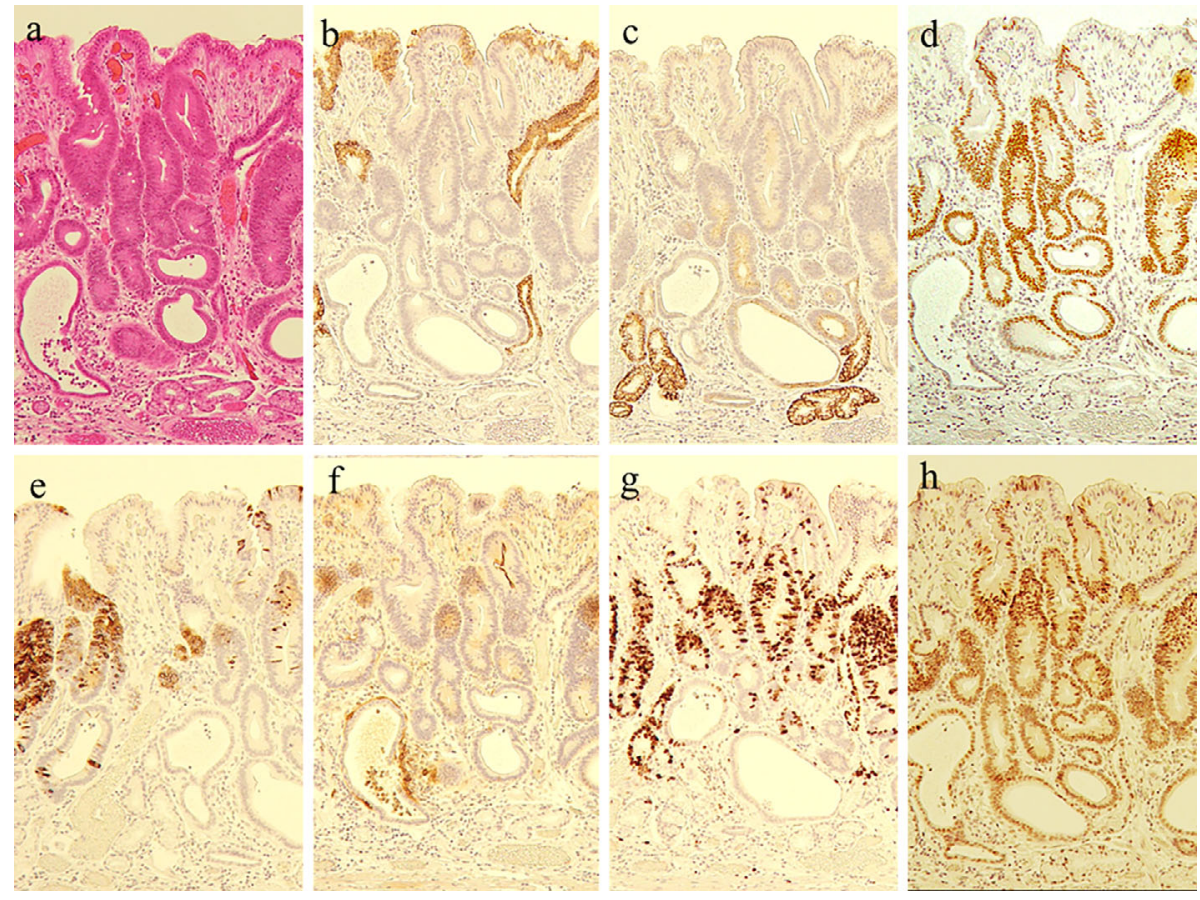

Figure 3. a: Hematoxylin and Eosin staining, b: MUC5AC, c: MUC6, d: CDX2, e: MUC2, f: CD10, g: Ki-67, h: p53. The neoplasm expressed CDX2, MUC2, and CD10, showing immunohistochemical characteristics suggestive of an intestinal mucin phenotype. Ki-67 was highly overexpressed (labeling index, $65.2 \%$ ), and p53 was diffusely highly overexpressed by neoplastic cells. 

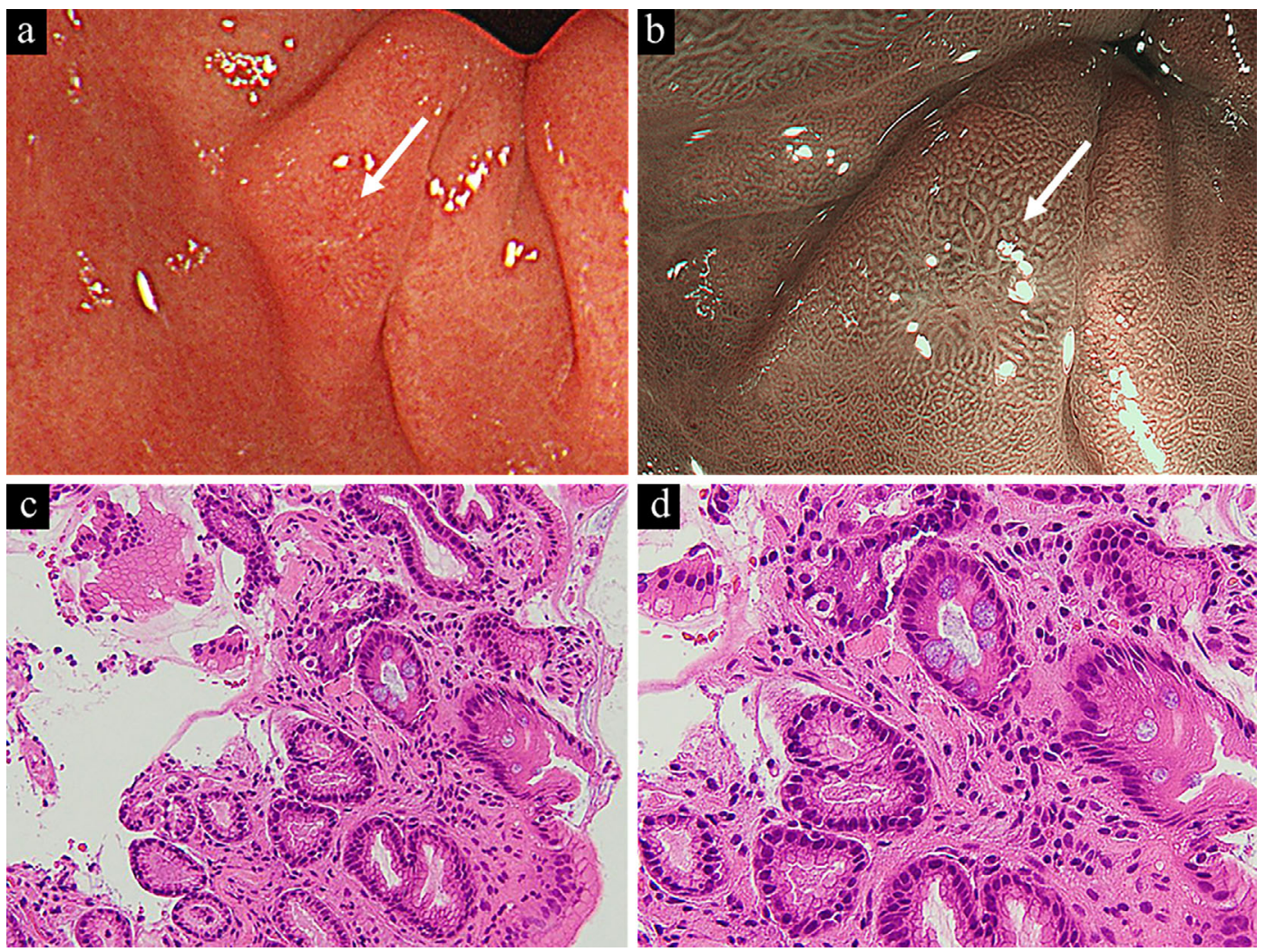

Figure 4. A small erosive lesion was detected in the prepyloric area as indicated by the white arrow (a), and narrow-band imaging with magnification endoscopy suggested benign inflammatory findings (b). A histologic examination of a biopsy specimen showed a non-neoplastic foveolar epithelium with intestinal metaplasia (c). Goblet cells were sporadically observed, as was mild stromal inflammation with fibrosis (d).

of intestinal metaplasia on $H$. pylori-uninfected gastric mucosa is theoretically required.

There have been three previous case reports of a solitary HPNGC with an intestinal mucin phenotype in the pyloric gland mucosa, but they did not report the coexistence of any intestinal metaplasia around the tumor (5-7). In this case, both neoplasms and intestinal metaplasia were observed in the pyloric gland mucosa with superficial gastritis. $H$. pylori-uninfected gastric mucosa occasionally accompanies the intestinal metaplasia according to higher concentrations of bile acid in the stomach (11), and it also occurs sporadically in the cardiac gland which closely resembles the pyloric gland both morphologically and functionally (12). The bile acid reflux may induce sporadic intestinal metaplasia in both glands without $H$. pylori infection, and it is also a well-known carcinogen for various gastrointestinal cancers (13). Consequently, we hypothesize that intestinal metaplasia arises in $H$. pylori-uninfected pyloric gland mucosa in response to chronic mucosal injury with bile acid reflux, leading to the possible onset of carcinogenesis, together with other factors including s smoking and drinking habit, and so on (14). Indeed, p53 was overexpressed by neoplastic cells in the present case as well as in other reported cases (5), thus suggesting multistage carcinogenesis.

Intestinal metaplasia has been thought to be a paracancerous lesion that coexists with the surrounding mucosa of the cancer (15). The possibility that intestinal metaplasia could also be a potential precancerous lesion has been demonstrated in animal experiments using $\mathrm{Cdx} 2$ transgenic mice who developed intestinal-type adenocarcinoma as well as intestinal metaplasia (16). Our case is demonstrated complete intestinal-type gastric cancer arising from non-atrophic gastric mucosa with sporadic intestinal metaplasia. Consequently, we hypothesize the possible carcinogenesis of this neoplasm to be via the occurrence of sporadic intestinal metaplasia. The accumulation of further cases with this histologic type of HPNGC is therefore required to fully confirm our hypothesis.

Two of three lesions were partially covered with a nonneoplastic foveolar epithelium which was positive MUC5AC and negative p53/Ki-67. The gastric cancer after $H$. pylorieradication shows similar histologic feature (17), but it is reportedly likely to express a gastric mucin phenotype (18). In this case, the neoplasm showed a pure intestinal mucin phenotype, and the background mucosa was diagnosed to be non-atrophic pyloric gland tissue based on the updated Sydney system. In addition, the patient had no H. pylorieradication history, and thus was diagnosed to be $H$. pyloriuninfected via various infection tests. Consequently, the possibility of spontaneous $H$. pylori elimination is quite unlikely.

This report suggests that HPNGC with an intestinal mucin 
phenotype has possibly been overlooked thus far in daily practice. The endoscopic findings closely resemble those of superficial gastritis, which is histologically caused by a superficial non-neoplastic foveolar epithelium covering the neoplastic ducts. Considering that advanced cases have not been reported thus far, this recently recognized histologic type of HPNGC may clinically appear to be slow-growing, like other types of HPNGC. As a result, solitary or multiple local lesions in the $H$. pyroli-uninfected pyloric gland mucosa should be closely observed using magnification endoscopy and also be histologically evaluated for any possible neoplastic characteristics.

The authors state that they have no Conflict of Interest (COI).

\section{References}

1. Ono S, Kato M, Suzuki M, et al. Frequency of Helicobacter pylori-negative gastric cancer and gastric mucosal atrophy in a Japanese endoscopic submucosal dissection series including histological, endoscopic and serological atrophy. Digestion 86: 59-65, 2012.

2. Matsuo T, Ito M, Takata S, Tanaka S, Yoshihara M, Chayama K. Low prevalence of Helicobacter pylori-negative gastric cancer among Japanese. Helicobacter 16: 415-419, 2011.

3. Tohda G, Osawa T, Asada Y, Dochin M, Terahata S. Gastric adenocarcinoma of fundic gland type: endoscopic and clinicopathological features. World J Gastrointest Endosc 8: 244-251, 2016.

4. Shibagaki K, Fukuyama C, Mikami H, et al. Gastric foveolar-type adenomas endoscopically showing a raspberry-like appearance in the Helicobacter pylori-uninfected stomach. Endosc Int 7: 784791, 2019.

5. Kotani S, Miyaoka Y, Fujiwara A, et al. Intestinal-type gastric adenocarcinoma without Helicobacter pylori infection successfully treated with endoscopic submucosal dissection. Clin J Gastroenterol 9: 228-232, 2016.

6. Ozaki Y, Suto H, Nosaka T, et al. A case of Helicobacter pylorinegative intramucosal well-differentiated gastric adenocarcinoma with intestinal phenotype. Clin J Gastroenterol 8: 18-21, 2015.

7. Yoshii S, Hayashi Y, Takehara T. Helicobacter pylori-negative early gastric adenocarcinoma with complete intestinal mucus phe- notype mimicking verrucous gastritis. Dig Endosc 29: 235-236, 2017.

8. Kimura K, Takemoto T. An endoscopic recognition of atrophic border and significance in chronic gastritis. Endoscopy 3: 87-97, 1969.

9. Dixon MF, Genta RM, Yardley JH, et al. Classification and grading of gastritis. The updated Sydney System. International Workshop on the Histopathology of Gastritis, Houston 1994. Am J Surg Pathol 20: 1161-1181, 1996.

10. Inoue M. Changing epidemiology of Helicobacter pylori in Japan. Gastric Cancer 1: 3-7, 2017.

11. Matsuhisa T, Arakawa T, Watanabe T, et al. Relation between bile acid reflux into the stomach and the risk of atrophic gastritis and intestinal metaplasia: a multicenter study of 2283 cases. Dig Endosc 25: 519-525, 2013.

12. Glickman JN, Wang H, Das KM, et al. Phenotype of Barrett's esophagus and intestinal metaplasia of distal esophagus and gastroesophageal junction: an immunohistochemical study of cytokeratin 7 and 20, Das-1 and 45 MI. Am J Surg Pathol 25: 87-94, 2001.

13. Liu $T$, Song $X$, Khan $S$, et al. The gut microbiota at the intersection of bile acids and intestinal carcinogenesis: an old story, yet mesmerizing. Int J Cancer 146: 1780-1790, 2020.

14. Karimi P, Islami F, Anandasabapathy S, Freedman ND, Kamangar F. Gastric cancer: descriptive epidemiology, risk factors, screening, and prevention. Cancer Epidemiol Biomarkers Prev 23: 700-713, 2014.

15. Hattori T. Development of adenocarcinomas in the stomach. Cancer 57: 1528-1534, 1986.

16. Mutoh H, Sakurai S, Satoh K, et al. Development of gastric carcinoma from intestinal metaplasia in Cdx2-transgenic mice. Cancer Res 64: 7740-7747, 2004.

17. Ito M, Tanaka S, Maeda M, et al. Role of the gastrin-gastrin receptor system in the expansive growth of human gastric neoplasms. Digestion 78: 163-170, 2008.

18. Yamamoto K, Kato M, Takahashi M, et al. Clinicopathological analysis of early-stage gastric cancers detected after successful eradication of Helicobacter pylori. Helicobacter 16: 210-216, 2011.

The Internal Medicine is an Open Access journal distributed under the Creative Commons Attribution-NonCommercial-NoDerivatives 4.0 International License. To view the details of this license, please visit (https://creativecommons.org/licenses/ by-nc-nd/4.0/).

(C) 2020 The Japanese Society of Internal Medicine Intern Med 59: 3155-3159, 2020 